Revista de la Escuela de Ciencias de la Educación, año 15, nRo. 14, vol. 2, JUlio a diciembre de 2019. PÁGinas 41-50. ISSN 1851-6297 (DESDE DICIEMBRE DE 2006 A DICIEMBRE DE 2017). ISSN 2362-3349 (EN LÍNEA). EL TRABAJO METODOLÓGICO EN EL CENTRO UNIVERSITARIO

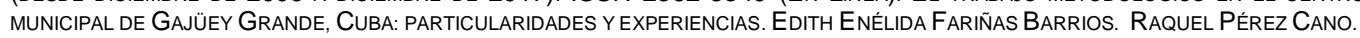

\title{
EL TRABAJO METODOLÓGICO EN EL CENTRO UNIVERSITARIO MUNICIPAL DE JAGÜEY GRANDE, CUBA: PARTICULARIDADES Y EXPERIENCIAS
}

\author{
Edith Enélida Fariñas Barrios* \\ Centro Universitario Municipal (CUM) de Jagüey Grande, Cuba \\ edith.farinas@umcc.cu \\ Raquel Pérez Cano* \\ Centro Universitario Municipal (CUM) de Jagüey Grande, Cuba \\ raquel.perez@umcc.cu
}

Recibido: 16/10/2018 Aceptado: 04/12/2018

\section{Resumen}

En los centros universitarios municipales (CUM) cubanos, el modelo pedagógico, el contexto local, el claustro pedagógico y la heterogeneidad de los estudiantes, son elementos a tener en cuenta para el desarrollo eficaz de sus procesos. En el artículo se analizan y describen particularidades y experiencias en la realización del trabajo metodológico como parte del proceso de formación continua de profesores de las carreras pedagógicas: Licenciatura en Educación preescolar y Licenciatura en Educación primaria en el CUM de Jagüey Grande. Los métodos teóricos de análisis, síntesis y generalización durante la revisión bibliográfica permitieron la determinación de los aspectos que constituyen posiciones de los autores. Los métodos empíricos de análisis de documentos y entrevista posibilitaron el estudio del tema a investigar.

Palabras clave: Formación continua - Trabajo metodológico - Centros universitarios municipales Particularidades - Experiencias.

\begin{abstract}
In cuban municipal university centers, the pedagogic model, the local context, the pedagogical cloister and the heterogeneity the students are elements to take into account for the efficient development of their processes. Particularities and experience of methodological work in the essay as part of continuous training process of professor of pedagogical careers: Bachelor of Preschool Education and Bachelor of Primary Education in these centers are analyzed and determined. The theoretical methods of analysis, synthesis and generalization during the bibliographic review allowed

\footnotetext{
* Profesora Auxiliar, MSc en Educación Superior, jefa de departamento de formación de pregrado en el CUM Jagüey Grande. En los últimos años desarrolló investigación sobre la implementación del trabajo metodológico en este centro, también sobre el proceso de formación de pregrado en estrecho vínculo con las instituciones locales.

* Profesora Auxiliar, MSc en Administración de empresa, directora CUM Jagüey Grande. En los últimos años desarrolló investigación sobre la implementación del trabajo metodológico en este centro, sobre el proceso de formación de pregrado en estrecho vínculo con las instituciones locales y la contribución al desarrollo local desde el CUM.
} 
Revista de la Escuela de Ciencias de la Educación, año 15, NRO. 14, vol. 2, JUlio a diciembre de 2019. PÁGinas 41-50. ISSN 1851-6297 (DESDE DICIEMBRE DE 2006 A DICIEMBRE DE 2017). ISSN 2362-3349 (EN LíNEA). El TRABAJO METOdOLÓGICO EN EL CENTRO UNIVERSITARIO

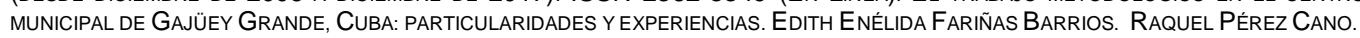

the determination of the aspects that constitute of the authors' positions. The empiric method of analysis of documents and interview made possible the study of the topic of research.

Keywords: Continuous training - Methodological work - Municipal university centers -Particularities Experiences.

\section{Introducción}

El artículo que presentamos contiene la valoración de ideas resultantes de la aplicación, desde el año 2012, de una estrategia dirigida a la formación continua de los profesores a través del trabajo metodológico, con acciones encaminadas a la planificación, ejecución y control del trabajo docente metodológico y científico metodológico en el Centro Universitario Municipal de Jagüey Grande, perteneciente a la Universidad de Matanzas, Cuba. En la universidad cubana, el trabajo metodológico constituye un sistema de acciones y actividades que se desarrollan a través de diferentes vías, con el propósito de garantizar la formación continua de los docentes y su contribución a la formación integral de los profesionales, "objetivo fundamental de la educación superior, lo que constituye un reto para directivos, profesores, estudiantes, instituciones educativas y otras vinculadas a este proceso" (Fraga, Milán y Echeverría, 2016, p. 275).

En relación con ello, ha sido necesario considerar transformaciones emanadas del Ministerio de Educación Superior: una de ellas, el proceso de integración de la Universidad cubana llevado a cabo en el 2014, trayendo consigo que la formación de los profesionales de la educación, anteriormente llevado a cabo en las universidades pedagógicas del país y rectoradas por el Ministerio de Educación, fuera transferida al Ministerio de Educación Superior. De esta forma, se responsabiliza a las universidades y, por consiguiente, a los CUM con la formación de maestros y profesores, pero no es hasta el curso escolar 2016-2017 que acceden estudiantes a estas carreras. La otra transformación significativa se produjo en este propio curso escolar, momento en que se inicia la implementación de los planes de estudio $\mathrm{E}$, con nuevas exigencias dirigidas a perfeccionar la formación de los profesionales, a partir de su concepción. Estos cambios repercutieron en el rediseño de la estrategia que se estaba ejecutando en aras de perfeccionar la formación continua de los profesores, en correspondencia con sus particularidades y cumplir con los objetivos propuestos.

Los centros universitarios municipales facilitan el acceso a las aulas universitarias de personas con formación precedente muy heterogénea, cuentan con un claustro único integrado por profesores a tiempo completo y por profesionales del territorio como profesores a tiempo parcial. Es importante contar con ellos por el conocimiento que poseen de los problemas de la localidad y la experiencia acumulada en su trabajo profesional que incorporan en su quehacer profesoral, pero al mismo tiempo resulta necesario realizar ajustes en el desarrollo del trabajo metodológico para garantizar la preparación pedagógica y didáctica eficiente de cada uno. Por otra parte, las particularidades del modelo pedagógico semipresencial deben ser tenidas en cuenta, ya que "la experiencia acumulada anteriormente es de estudios presenciales" (Horruitiner, 2006, p. 34).

Al respecto, se hace necesaria la preparación del profesor, al reconocer el rol esencial que su desempeño tiene en el éxito institucional para la gestión y desarrollo de los procesos universitarios y su incidencia en la calidad del egresado en el contexto municipal, si se tiene en cuenta que en los reglamentos se traza la política, la organización general, pero existen particularidades de este nivel de dirección subordinado a la universidad que demandan la búsqueda de variadas vías en aras de alcanzar la calidad en la formación continua de los docentes, de ahí que el objetivo del presente artículo consiste en: exponer particularidades y experiencias de las autoras en torno a la realización del trabajo metodológico como parte de la formación continua de los profesores de carreras pedagógicas en el Centro Universitario Municipal de Jagüey Grande.

El artículo se sustenta en entrevistas a profesores y el análisis de documentos resultantes de la planificación, ejecución, control y evaluación del sistema de trabajo metodológico; asimismo los métodos teóricos: análisis, síntesis y generalización durante la revisión bibliográfica y procesamiento de la información; permitieron la determinación de los aspectos que se exponen.

\section{La formación continua del profesor: prioridad para la educación superior}

En diferentes eventos y debates públicos: mundiales y regionales, celebrados desde la última década de la pasada centuria sobre educación superior, se ha considerado como paradigma de la nueva universidad educación superior de alta calidad para todos durante toda la vida, se ha defendido el principio de la educación superior como bien público social en estrecha relación con los conceptos de calidad, pertinencia y responsabilidad social, en vínculo directo con el contexto en que tiene lugar el proceso de formación y se ha significado la preparación del profesor para lograrlo. 
Revista de la Escuela de Ciencias de la Educación, año 15, nRo. 14, vol. 2, JUlio a diciembre de 2019. PÁGinas 41-50. ISSN 1851-6297 (DESDE DICIEMBRE DE 2006 A DICIEMBRE DE 2017). ISSN 2362-3349 (EN LÍNEA). EL TRABAJO METODOLÓGICO EN EL CENTRO UNIVERSITARIO

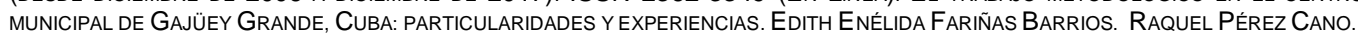

La Organización de las Naciones Unidas para la Educación, la Ciencia y la Cultura (UNESCO), en la Conferencia Mundial sobre Educación Superior:

La educación superior en el siglo XXI: Visión y acción aprobó, como elemento esencial para las instituciones de enseñanza superior, la necesidad de la política de formación del personal y de establecer directrices claras sobre los docentes para enseñar a sus alumnos a aprender y a tomar iniciativas. Se señala la importancia de tomarse medidas adecuadas en materia de investigación, así como de actualización y mejora de sus competencias pedagógicas mediante programas adecuados de formación del personal que estimulen la innovación permanente en los planes de estudio y los métodos de enseñanza y aprendizaje (...) a fin de garantizar la excelencia de la investigación y la enseñanza (UNESCO, 1998, p. 4).

En fecha reciente, la Oficina Regional de Educación para América Latina y el Caribe (OREALC/UNESCO, 2017) convocó la Reunión Regional de Ministros de Educación de América Latina y el Caribe "E2030: Educación y Habilidades para el siglo 21", en Buenos Aires, Argentina donde se propone como acuerdo:

Nos comprometemos a fortalecer los programas de formación docente inicial y continua, con atención especial en la planificación contextualizada de las ofertas, la revisión de los contenidos y planes de estudio y la preparación permanente de los formadores, a fin de garantizar propuestas formativas innovadoras que preparen, motiven (...) a los docentes y directivos para los desafíos y oportunidades que presenta la enseñanza en el siglo 21. Avanzaremos en políticas orientadas a sentar las condiciones adecuadas para que la docencia se transforme en una profesión de excelencia, asegurando la participación de los docentes y otros profesionales de la educación en su diseño, implementación, monitoreo y evaluación (OREALC/UNESCO, 2017, pp.11-12).

De este modo, al analizar la formación continua de los profesores que forman los profesionales de la educación, constituye prioridad y meta para los gobiernos latinoamericanos y, por tanto, para las universidades, la formación de maestros calificados.

En Cuba, el Ministerio de Educación Superior, en correspondencia con las demandas de la sociedad, ha ofrecido especial atención a la formación continua de los docentes para enfrentar la educación que reclama esta época, demostrado en las conferencias ofrecidas por los ministros de Educación Superior: Rodolfo Alarcón Ortiz en el Congreso Universidad 2014 y José Ramón Saborido Loidi en Universidad 2018. Alarcón expresó: "Es imprescindible que hagamos todo el esfuerzo posible por elevar al máximo el compromiso, nivel académico y científico de nuestros profesores" (Alarcón, 2014, p. 7). Saborido (2018) ratifica esta idea al afirmar que "garantizar en calidad y cantidad la formación de profesores", constituye un desafío para la Universidad y que las políticas públicas "deben favorecer la formación y superación de maestros y profesores" (Saborido, 2018, p. 15).

En efecto, la formación continua es necesaria para los profesionales de cualquier área de conocimiento y para los profesionales de la educación resulta esencial. En este sentido, constituye una preocupación esencial en el contexto político, económico, tecnológico y sociocultural actual abordándola desde un enfoque interdisciplinario que contribuya a problematizar los contenidos y el logro de óptimos resultados en los estudiantes.

En la Universidad cubana, la conjugación armónica y sistémica del postgrado, la investigación educativa y el trabajo metodológico contribuyen a la formación continua del profesorado. Sostenemos que este último resulta de gran significación para perfeccionar su desempeño, reforzar la formación científica y pedagógica, dirigir la atención a la creatividad e independencia en la gestión de los colectivos metodológicos y fortalecer su compromiso con la función que realizan. Es de destacar la prioridad ofrecida por los directivos del MES, tal es así que en el Congreso Internacional Pedagogía 2015, Alarcón planteó: "debemos prestar mucha atención al trabajo metodológico sistemático" (Alarcón, 2015, p. 6).

El trabajo metodológico, según el artículo 17 del Reglamento para el Trabajo Docente y Metodológico en la educación superior es:

La labor que, apoyados en la Didáctica, realizan los sujetos que intervienen en el proceso docente educativo, con el propósito de alcanzar óptimos resultados en dicho proceso, jerarquizando la labor educativa desde la instrucción, para satisfacer plenamente los objetivos formulados en los planes de estudio (...) Las formas fundamentales del trabajo metodológico son: docente-metodológico y científico-metodológico; están estrechamente vinculadas entre sí y 
Revista de la Escuela de Ciencias de la Educación, año 15, NRO. 14, vol. 2, JUlio a diciembre de 2019. PÁGinas 41-50. ISSN 1851-6297 (DESDE DICIEMBRE DE 2006 A DICIEMBRE DE 2017). ISSN 2362-3349 (EN LíNEA). El TRABAJO METOdOLÓGICO EN EL CENTRO UNIVERSITARIO

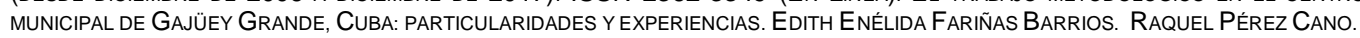

en la gestión del trabajo metodológico deben integrarse como sistema en respuesta a los objetivos propuestos (MES, 2018, p.6).

Asimismo, se realiza tanto de forma individual como colectiva y garantiza el desarrollo con calidad del proceso docente educativo.

Cuando hablamos de garantizar calidad tenemos que ser consecuentes con el contexto y el rol de la educación, por ser un proceso que debe adaptarse a su época, a la necesidad de considerar el desarrollo tecnológico y a la celeridad en la producción de conocimientos en las diferentes áreas. Desde esa perspectiva, se valora la localidad como escenario fundamental para el desarrollo de un trabajo metodológico que se revierta en mejor desempeño del profesor y garantice el desarrollo eficaz del proceso de formación, pudiendo integrar la docencia con lo laboral y lo investigativo, de manera que se consiga como resultado la calidad del profesional que egresa, sin perder de vista los procesos mundiales, regionales, nacionales, provinciales y su incidencia en lo local.

Como parte del proceso de universalización de la educación superior, iniciado en 1962, se trabajó por extender la universidad y sus procesos sustantivos a toda la sociedad, de ahí que como continuidad de este proceso, en el 2002, tiene lugar la municipalización de la educación superior y las aulas universitarias se abren en cada localidad del país con la creación de las sedes universitarias municipales, actuales filiales y centros universitarios municipales, asegurando el acceso a la educación terciaria y el egreso de profesionales comprometidos con el desarrollo local, capaces de responder a las demandas del territorio para potenciar el desarrollo científico tecnológico, económico y social.

En el artículo 9 del Reglamento de trabajo docente y metodológico se plantea: El centro universitario municipal es el nivel de dirección que tiene básicamente un carácter local en la dinámica de su desempeño, al frente del cual está el director como autoridad máxima, quien se subordina directamente al rector. Es una unidad organizativa de la universidad que tiene como objetivo llevar a cabo todos los procesos y funciones sustantivas de la Educación Superior en la magnitud que se demande por el municipio y en la medida que se asegure la calidad requerida. (MES, 2018, p.4).

En el CUM la concepción del trabajo metodológico se corresponde con los aspectos generales planteados en los documentos normativos del MES, sin embargo, su carácter local unido a otras particularidades, demandan el análisis acerca de cómo proceder para garantizar efectividad en la del trabajo metodológico que se revierta en la preparación eficaz del personal docente.

Siguiendo la idea anterior y considerando que los profesores son el recurso humano más valioso para suscitar cambios y contribuir al desarrollo de la sociedad, su formación es esencial para lograr la eficacia del sistema educativo, por tanto, un aspecto relevante a considerar es la composición y características distintivas del claustro pedagógico en el CUM, integrado por profesores a tiempo completo y a tiempo parcial, estos últimos profesionales de reconocido prestigio y resultados relevantes en la labor que desempeñan en la localidad que les permite aportar ideas al desarrollo del trabajo metodológico y del proceso docente educativo sobre su experiencia práctica, habilidades y destrezas propias de su profesión. Sin embargo, esto implica adecuar la planificación, ejecución y control de las actividades metodológicas que se realizan a las condiciones de este personal con doble vínculo laboral.

En este sentido, la Dirección de Formación del Profesional del Ministerio de Educación Superior considera que la formación de profesionales en los municipios implica un cambio de mentalidad en todos, incluyendo a los directivos de los territorios que "exigen una transformación radical de los objetivos, los métodos y los contenidos de nuestros planes y programas, de nuestras clases, de nuestro papel en el proceso docente para situar al estudiante en el centro de atención" (Alarcón, 2015, p. 3). Se resalta la importancia de reforzar el trabajo metodológico dirigido a fortalecer el proceso de formación en estos centros, como unidad organizativa de la universidad que tiene carácter local. Se enfatiza en la necesidad de asumir una posición más activa en las carreras de las ciencias pedagógicas para que estos profesionales sean mejores en el cumplimiento de sus funciones.

Por ende, lograr eficiencia y eficacia en el trabajo metodológico, requiere la disposición y preparación profesional del profesorado para participar activa y reflexivamente en las diferentes actividades, desarrollar habilidades profesionales para la dirección del proceso docente educativo, más aún cuando la modalidad semipresencial, mediante la cual se realiza el proceso de formación, posee elementos novedosos en comparación con las prácticas educativas tradicionales, tales como: el desarrollo de la clase encuentro, la orientación y control de las actividades a desarrollar por el estudiante durante la autopreparación como forma de organización primordial en este modelo pedagógico, por lo que "se precisa una transformación en el desempeño profesional pedagógico que conduce a la problemática de la educación de los educadores, al requerimiento de una cultura profesional que matice la expresión del quehacer cotidiano de los docentes" (Martín, 2015, p. 340).

Por otra parte, en el modelo de formación de la Universidad cubana se establece que: 
Revista de la Escuela de Ciencias de la Educación, año 15, nRo. 14, vol. 2, JUlio a diciembre de 2019. PÁGinas 41-50. ISSN 1851-6297 (DESDE DICIEMBRE DE 2006 A DICIEMBRE DE 2017). ISSN 2362-3349 (EN LíNEA). El TRABAJO METOdOLÓGICO EN EL CENTRO UNIVERSITARIO

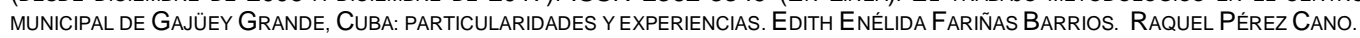

Lo metodológico es inherente a todos los procesos que tienen lugar en la educación superior. No es privativo de la labor de formación, como inicialmente se ha entendido e incluso se ha incorporado a nuestras reglamentaciones vigentes. Es necesaria una labor metodológica propia del quehacer investigativo y extensionista, en respuesta a las particularidades de cada uno de ellos, al igual que ocurre con el proceso de formación (Hourruitiner, 2006, pp. 54-55).

Es así que las consideraciones anteriores permiten plantear experiencias sobre cómo desarrollar el trabajo metodológico en el centro universitario municipal como vía para la formación continua de profesores, según las peculiaridades de esta unidad organizativa y del establecimiento de vínculos con las instituciones educativas y productoras de conocimientos de la localidad, que favorezcan la calidad de los procesos universitarios y se revierta en la formación de un profesional integral.

\section{El trabajo metodológico como parte de la formación continua de profesores en el CUM Jagüey Grande}

Desde el año 2012, en el CUM de Jagüey Grande se han diseñado acciones de superación del claustro de profesores, atendiendo a 3 direcciones: superación de posgrado, investigación pedagógica y trabajo metodológico. En lo referente al trabajo metodológico la estrategia elaborada contiene acciones en dos direcciones: trabajo docente metodológico y trabajo científico metodológico. Como parte del trabajo docente metodológico se ha planificado y ejecutado en cada curso escolar el sistema de actividades metodológicas, las que han constituido espacios y alternativas para el intercambio sistemático de saberes y experiencias en temas variados, así como del trabajo científico metodológico (Rodríguez, Fariñas y Pérez, 2015).

En este artículo nos vamos a focalizar en las experiencias para desarrollar el trabajo metodológico en el Centro Universitario Municipal como parte de la formación continua de los docentes, acorde con las particularidades de este nivel de dirección. Ahora bien, ¿cuáles son estas particularidades?, ¿cómo se ha procedido?, ¿qué experiencias podemos compartir?

Para poder lograr un óptimo resultado en la formación del profesor recaba determinar el estado real de su preparación, de forma tal que se proyecten acciones certeras, por ello el diagnóstico de necesidades y potencialidades de los docentes ha constituido el punto de partida para el diseño y ejecución de la estrategia. Como proceso continuo y sistemático, se ejecuta a través del intercambio sistemático con el profesor y del seguimiento a las tareas que realiza, tales como: control a actividades docentes, de investigación y extensión, de su participación activa en actividades metodológicas, las que han facilitado la determinación de necesidades de aprendizaje para la dirección del proceso de formación en pregrado y posgrado. Debemos precisar que los profesores a tiempo parcial, son profesionales de la educación que se desempeñan como directivos o metodólogos en la Dirección Municipal de Educación y, por tanto, están relacionados directamente con los centros docentes -círculos infantiles y escuelas primarias- donde laboran los estudiantes del CUM.

Los resultados del diagnóstico constituyen referencia para la elaboración de los planes individuales de cada profesor y del sistema de trabajo metodológico. Estos incluyen: la elaboración de la estrategia metodológica del CUM, de los planes metodológicos de departamentos y carreras elaborados para cada curso escolar, los que se complementan e interrelacionan con la planificación y ejecución de acciones individuales e incluyen las dos formas fundamentales del trabajo metodológico: docente-metodológico y científico-metodológico. La estrategia y los planes no son rígidos, se modifican y actualizan a partir de los resultados, los análisis y las valoraciones que se realizan, según los plazos establecidos en los diferentes niveles organizativos y de dirección, posibilitando un trabajo sistemático y enriquecedor de la práctica pedagógica.

La estrategia y el plan metodológico contienen la programación para la preparación sistemática, donde se proyectan acciones colectivas que demandan responsabilidades individuales, dirigidas no solo a que el profesor perfeccione su nivel de preparación a partir del tratamiento a las debilidades detectadas, sino también a que socialice sus saberes y experiencias positivas como resultado de su labor, acorde con las fortalezas diagnosticadas y lo dote de "una formación profesional sólida que le permita participar directamente en los distintos procesos de mejora y toma de decisiones que acontecen en torno a la práctica pedagógica" (Nava y Reynoso, 2014, p.140).

Sobre esta base de análisis, al considerar al profesor como un recurso humano esencial, se enfatiza en la responsabilidad social de la universidad, en la pertinencia social, en la importancia de formar a los profesionales de la educación en estrecho vínculo con las características socioculturales y económicas del contexto, con la preparación óptima para contribuir al desarrollo local, aspecto esencial en el logro de la calidad y que se convierte en exigencia a tener en cuenta. Por tanto, es necesario considerar que el centro universitario municipal es uno de los actores de la educación superior involucrado en el desarrollo local y "debe actuar como eje local aglutinador de patrimonio humano e innovativo de la localidad y como agente del conocimiento y la innovación”. (Núñez y Alcázar, 2016, p. 200). Esta visión ha constituido un reto en la 
Revista de la Escuela de Ciencias de la Educación, año 15, NRO. 14, vol. 2, JUlio a diciembre de 2019. PÁGinas 41-50. ISSN 1851-6297 (DESDE DICIEMBRE DE 2006 A DICIEMBRE DE 2017). ISSN 2362-3349 (EN LíNEA). El TRABAJO METOdOLÓGICO EN EL CENTRO UNIVERSITARIO

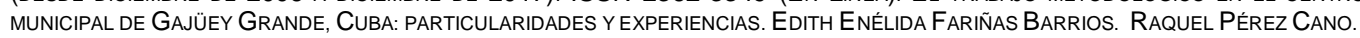

preparación de los profesores, por lo que ha sido necesario desarrollar una labor metodológica dirigida al perfeccionamiento de la investigación y la extensión, acorde a las particularidades de estos procesos en interacción con el de formación.

De este modo, se acentúa la responsabilidad social de la institución, en la pertinencia social, en la importancia de formar integralmente a los profesionales de la educación en estrecho vínculo con las características socioculturales y económicas del contexto, con la formación vocacional, el desarrollo de sus modos de actuación y de las competencias necesarias para solucionar los problemas de la profesión docente y contribuir al desarrollo de la localidad, aspecto cardinal en el logro de la calidad en los centros educacionales.

Por tanto, el sistema de trabajo metodológico ha estado dirigido al perfeccionamiento de la labor docente y al proceso formativo de los profesores, desde la práctica pedagógica en los diferentes escenarios educativos, en interacción con las instituciones de la localidad, fundamentalmente en los centros escolares donde los estudiantes desarrollan su labor docente. A partir del intercambio con los directivos de estos centros se ejecutan actividades que preparan al profesor para integrar los componentes: académico, laboral e investigativo del proceso de formación en las condiciones concretas donde se desempeñan los profesionales en formación, con el propósito de adecuar las tareas docentes a la realidad concreta, pudiendo contribuir a satisfacer la demanda de la sociedad.

Visto de este modo, en el trabajo metodológico, con y por los profesores, se ha tenido en cuenta el nexo teoría - práctica pedagógica, es decir, se les ha otorgado un rol activo en el cumplimiento de sus funciones en relación con el contexto educativo local. De ahí que se han estrechado vínculos con otros colegas y directivos de los centros educacionales donde los profesionales en formación desempeñan su labor docente, así como con otros factores del municipio relacionados con el sistema educacional. Así, el profesor universitario, como formador de formadores se prepara para desarrollar un "proceso docente educativo alternativo, coordinado, creativo y desarrollador. Permite, además, elevar la formación del potencial humano que participa en él" (Miqueli y Polo, 2017, p. 43).

Es por ello que las estrategias y planes elaborados contienen actividades y acciones encaminadas a perfeccionar los modos de actuación del docente que generen impacto en el aprendizaje de los estudiantes, a partir del nexo de la teoría con la práctica y su contribución al fortalecimiento de la profesión, a partir de potenciar la autoevaluación de cada profesor y que cada cual sea un ente activo en la evaluación de las actividades que se ejecutan. De ahí que hemos trabajado en preparar y actualizar a los docentes sistemáticamente en temas: pedagógicos, didácticos, metodológicos, psicológicos, políticos, económicos, jurídicos, sociales y en conocimientos en sus respectivas ramas del saber a través de actividades individuales y colectivas que perfeccionen su labor docente.

Por otra parte, establecer la relación estrecha entre el trabajo metodológico individual y colectivo es vital para asumir las responsabilidades individuales. Resulta necesario el estudio individual previo a la actividad colectiva planificada con el propósito de que en este momento se socialice y enriquezca el punto de vista de cada uno, se valore la interpretación realizada en lo individual para poder llegar a consenso sobre cuáles son las prácticas más eficaces que favorecen la diversificación de los modos de actuar y apropiarse de aquellos procedimientos que se adaptan a las particularidades de cada profesor, de los estudiantes y de la asignatura que imparte.

En estos espacios de intercambios se ha utilizado el aprendizaje colaborativo en la planificación, elaboración y solución a problemas propios de la profesión pertinentes al contexto local. Estos análisis se desarrollan a través de: preparación en el colectivo de la carrera, de disciplina y de asignatura; reunión metodológica; clase metodológica, instructiva y demostrativa; clase abierta; de comprobación y taller metodológico. Se establecen debates de los resultados de investigaciones de los profesores como parte de su labor didáctica y metodológica y del trabajo científico metodológico en seminarios científico metodológicos.

Se sostiene que "la formación del docente debe ir pareja a los cambios didácticos y socioeducativos que se van generando en cada momento" (Vázquez, 2015, p. 150). Por lo tanto, se ha tenido en cuenta la adecuación a las particularidades del modelo pedagógico semipresencial, el que con su puesta en práctica favorece cumplir los propósitos del pleno acceso, la continuidad de estudios de los ciudadanos, al facilitar que el estudiante pueda combinar su formación como universitario con la actividad laboral que desempeña. Estas características demandan actitudes pedagógicas y didácticas diferentes a las tradicionales de la enseñanza presencial para que motiven al estudiante y asuma una actitud responsable ante el estudio, consiguiendo autorregular su aprendizaje.

Por ello, se han encauzado de manera planificada, organizada y en consonancia con las necesidades de los profesores y las particularidades del modelo pedagógico, actividades dirigidas a potenciar su desempeño de manera acertada en este contexto, las que a su vez demandan el intercambio, la reflexión, el análisis y la demostración en temas tratados, tales como: la estructura metodológica de la clase encuentro, como tipo de clase fundamental en esta modalidad; métodos de enseñanza aprendizaje; orientación, control y evaluación del estudio independiente; la demostración de variadas estrategias de 
Revista de la Escuela de Ciencias de la Educación, año 15, nRo. 14, vol. 2, JUlio a diciembre de 2019. PÁGinas 41-50. ISSN 1851-6297 (DESDE DICIEMBRE DE 2006 A DICIEMBRE DE 2017). ISSN 2362-3349 (EN LÍNEA). EL TRABAJO METODOLÓGICO EN EL CENTRO UNIVERSITARIO

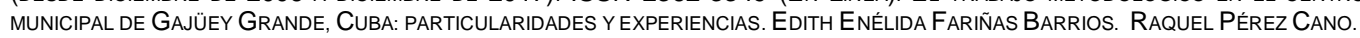

enseñanza y aprendizaje; potencialidades y uso de las tecnologías de la informática y las comunicaciones; implementación de las estrategias curriculares; así como la formación y desarrollo de valores.

Actualmente, para contrarrestar el decrecimiento en el ingreso a los cursos por encuentros y a distancia que tuvo lugar en el período 2009-2015, dado por la casi nula presentación a exámenes de ingreso, se ofrece la opción de cursar y aprobar las asignaturas que constituyen requisitos de ingreso a la educación superior en el primer año de la carrera, por tanto se incluyeron en el plan de estudio como asignaturas: Matemática Básica, Español Básico e Historia de Cuba Básica. De esta forma se da continuidad a la política trazada por el MES en aras de garantizar el acceso a las aulas universitarias e ingresan a los CUM personas con formación previa heterogénea, las que no tuvieron la posibilidad de acceder a los cursos diurnos y, en muchos casos, desvinculados de la actividad de estudio. Estas peculiaridades, unidas a las características personológicas de cada estudiante, inciden en la constitución de grupos muy diversos, que requieren ofrecer atención individualizada a sus miembros y les proporcionen las ayudas necesarias para vencer insuficiencias precedentes y contribuir a su formación como profesionales.

Asimismo a las carreras pedagógicas que se desarrollan en el centro: Licenciatura en Educación preescolar y Licenciatura en Educación primaria, ingresan estudiantes de fuentes diversas: egresados de escuelas pedagógicas de nivel medio que poseen una formación inicial en la profesión, estos son jóvenes que continúan sus estudios en este tipo de curso de forma directa y se desempeñan como maestros y profesores en los centros educativos del municipio; también acceden personas que no están vinculadas laboralmente a la docencia y otras que han iniciado recientemente su labor como maestros o asistentes educativas. Estos últimos graduados de diferentes centros y enseñanzas: preuniversitarios, educación de adultos o politécnicos. Las diferencias se agudizan en los estudiantes que durante cinco o más años han permanecido desvinculados de la actividad de estudio.

Lo antes expuesto conduce a expresar que las particularidades del modelo pedagógico demandan "la búsqueda de respuestas educativas que faciliten el aprendizaje de todo el alumnado en su diversidad y la preparación de los profesores (...) como aspecto esencial (...) que permita una formación profesional de calidad para todos" (Pichs, Hernández y Benítez, 2006, p. 293). Se trata entonces, de tener en cuenta que "la atención a la diversidad resulta todo un reto, especialmente en educación superior" (Amaro, Méndez, y Mendoza, 2015, p. 200), y, por tanto, una exigencia para la proyección y ejecución del trabajo metodológico.

En consecuencia se han desarrollado actividades metodológicas a nivel de CUM, departamentos docentes, carreras y colectivos de año dirigidas a la preparación del profesor, tanto en lo referente al estudio y análisis de la teoría relacionada con el tratamiento a la diversidad como en la aplicación práctica con el propósito de ponerlo en condiciones de ofrecer ayuda personalizada a los estudiantes a través de la elaboración, orientación, control y evaluación de las guías de estudio, de las tareas docentes, de la demostración de variadas estrategias de aprendizaje y de cómo potenciar la autovaloración, autocontrol y autorregulación del aprendizaje que eleven su autoestima y le concedan seguridad y responsabilidad ante el estudio.

Teniendo esto en cuenta, se ha potenciado la reflexión del profesor sobre sus estrategias de enseñanza y de aprendizaje que lo preparen para enseñar la autorregulación del aprendizaje, demostrar variadas estrategias de aprendizaje al abordar los contenidos, siendo el encargado de organizar situaciones de enseñanza aprendizaje encaminadas a enseñar y demostrar las posibles estrategias que pueden ser utilizadas para que cada estudiante aprenda a decidir y conformar las suyas. "Esto implica una formación en valores y una nueva actitud ante el saber, con el pleno ejercicio de la creatividad, la independencia pedagógica, la capacidad de autoperfeccionamiento, de investigación..." (Martín, 2015, p. 341).

A tono con lo anterior, ha sido necesario preparar al profesor para que reflexione sistemáticamente sobre: el proceso docente educativo, sus potencialidades y debilidades para aprender y enseñar, la aplicación de la ciencia y la tecnología, como adecuar las experiencias de los otros a sus características, las de los estudiantes y las de su asignatura, de manera que potencie su crecimiento como profesional con pensamiento crítico, con una concepción humanista, dotado de competencias para formar educadores con sólidos valores, actitudes, comprometido con la profesión, con el desarrollo de la educación cubana y su repercusión en el desarrollo social. Un profesor reflexivo, analítico con una formación interdisciplinaria y transdisciplinaria constituye un patrón de referencia para sus estudiantes en el desarrollo del sistema de conocimientos, de sus valores y actitudes, de forma tal que se responsabilice con el análisis del contexto socioeconómico, político e histórico y la transformación de su práctica, aspecto resulta necesario mantener un seguimiento que garantice la formación eficaz de los profesionales.

Desde esta perspectiva el trabajo metodológico, como proceso de gestión de la pedagogía y la didáctica favorece la preparación del colectivo de profesores para aprender a aprender, aprender a enseñar y enseñar a aprender, de manera que con su actuar pedagógico constituya un modelo en la formación de un profesional de la educación en consonancia con las exigencias de la sociedad, capaz de asumir un rol transformador y proactivo "comprometido con la ciencia, y que reconozca la formación científica como una vía estratégica para la generación de conocimientos” (Rodríguez, Reyes y Pérez, 2017, p. 101). 
Revista de la Escuela de Ciencias de la Educación, año 15, NRO. 14, vol. 2, JUlio a diciembre de 2019. PÁGinas 41-50. ISSN 1851-6297 (DESDE DICIEMBRE DE 2006 A DICIEMBRE DE 2017). ISSN 2362-3349 (EN LíNEA). El TRABAJO METOdOLÓGICO EN EL CENTRO UNIVERSITARIO

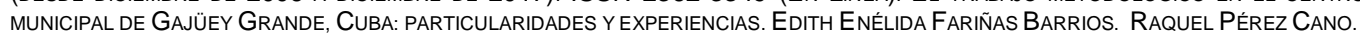

Como parte del trabajo científico metodológico cada curso escolar se desarrolla el seminario científico que constituye un espacio donde los profesores intercambian sus experiencias y resultados de la actividad investigativa en líneas y temas pedagógicos. Las experiencias con mejores resultados son llevadas a la conferencia científico metodológica que se desarrolla a nivel de Universidad.

El trabajo metodológico como parte de la formación continua requiere motivación, compromiso y disposición que favorezca la elevación del nivel cultural, científico, pedagógico del profesor y el desarrollo de habilidades para manejar pedagógicamente las tecnologías, lo que constituye una cualidad necesaria en la formación permanente del personal docente. El profesor debe poseer habilidades para la búsqueda y procesamiento de información actualizada, el trabajo en redes, el trabajo con las plataformas interactivas y la elaboración de medios variados, énfasis especial en los CUM, donde ocupa un lugar clave la autopreparación del estudiante y la mayor parte de la bibliografía actualizada se encuentra en formato digital. En las diferentes actividades metodológicas realizadas se opera con las tecnologías, lo que contribuye a preparar al profesor para desarrollar un proceso educativo mediado por las TIC.

Ahora bien, concebir el sistema de trabajo metodológico, implica considerar el nexo estrecho con los diferentes niveles de dirección en los que se desarrolla el trabajo metodológico en la Universidad de Matanzas, como centro de educación superior al que pertenecemos y donde los profesores participan como vía para perfeccionar su formación a nivel de facultad, colectivos de carreras, colectivos de disciplina y asignaturas, lo que requiere el intercambio sistemático con los profesores que conducen cada uno de estos niveles organizativos y sus miembros. Es de destacar que, aunque mensualmente se desarrollan actividades metodológicas centralizadas para la atención a los profesores de los CUM y las filiales universitarias, el intercambio sistemático se mantiene a través de los medios de comunicación.

Es importante enfatizar que, con sistematicidad mensual se efectúa reunión de análisis y control del trabajo donde se valoran los resultados de la etapa, el cumplimiento de las actividades según lo planificado, estos constituyen balances parciales, a partir de los cuales se divulgan las experiencias positivas de los departamentos y colectivos de carrera que sirven de referencia para rediseñar la estrategia. Al concluir el primer semestre y el curso escolar se realiza la valoración del cumplimiento de las acciones, de los resultados del profesor y de los estudiantes. El estado de cumplimiento del trabajo metodológico forma parte de la evaluación profesoral y constituye la base para el rediseño del sistema.

\section{Algunos criterios emitidos por los profesores en entrevistas realizadas}

Con respecto a los fundamentos psicopedagógicos del modelo pedagógico y la estructura metodológica de la clase encuentro, los profesores refieren:

Profesor a tiempo parcial: "En las actividades metodológicas que hemos participado se nos ha posibilitado apropiarnos de la didáctica de la clase encuentro, de la terminología a utilizar en la educación superior y, en particular de las características de la modalidad semipresencial, que no es a lo que nos enfrentamos sistemáticamente".

Profesor a tiempo parcial: "Ajustar el horario para que los profesores que trabajamos en la Dirección Municipal de Educación podamos participar nos permite apropiarnos de lo que se debate y aportar nuestros criterios".

Profesor a tiempo completo: "Creo que haber tenido en cuenta, en las clases metodológicas: instructivas y demostrativas, así como en las clases abiertas, el tratamiento a la estructura metodológica de la clase encuentro, la orientación y control del estudio independiente y el nexo educación - instrucción, aunque no constituya el objetivo de la actividad, ha contribuido al mejor desarrollo de la docencia que impartimos".

Concerniente al establecimiento de vínculos e intercambios con directivos y docentes de los centros escolares para perfeccionar su práctica pedagógica, apropiarse de los problemas existentes y contribuir al perfeccionamiento de la docencia y al desarrollo local expresan:

Profesor a tiempo completo: "Realizar actividades metodológicas en los círculos infantiles, intercambiar con educadoras nos ha familiarizado con la realidad de estos centros donde imparten actividades educativas nuestras estudiantes, eso nos ayuda a que podamos establecer un vínculo más fuerte y buscar solución a los problemas reales a los que se enfrentan en su labor educativa".

Profesor a tiempo parcial: "Analizar nuestros problemas reales y prepararnos para solucionarlos es importante, algo positivo es que a nivel de carrera intercambiamos y buscamos la mejor forma para planificar e impartir las clases, asimismo la reflexión individual que realizamos a partir de lo analizado colectivamente y su adecuación a las características de los estudiantes y a la de cada profesor".

Profesor a tiempo completo: "Con respecto a la necesidad de contribuir al desarrollo local desde la clase, creo que hemos avanzado en lo referente a la relación con las escuelas para la determinación de las líneas y temas de investigación y para la proyección de la extensión desde las asignaturas del currículo, sin embargo, debemos buscar otras vías para seguir preparándonos, pues aún no se utilizan todos los recursos encaminados a lograr la integración entre estos procesos que garantice la formación integral del docente en formación y su influencia en la transformación de la localidad". 
Revista de la Escuela de Ciencias de la Educación, año 15, nRo. 14, vol. 2, JUlio a diciembre de 2019. PÁGinas 41-50. ISSN 1851-6297 (DESDE DICIEMBRE DE 2006 A DICIEMBRE DE 2017). ISSN 2362-3349 (EN LÍNEA). EL TRABAJO METODOLÓGICO EN EL CENTRO UNIVERSITARIO

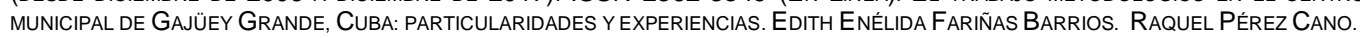

Referente al desarrollo de actividades metodológicas dirigidas a que los profesores la atención diferenciada para lograr una adecuada atención a sus necesidades formativas, en correspondencia con la heterogeneidad de los estudiantes, consideran que persisten insuficiencias, algunos expresan: "debemos continuar preparándonos", "es necesario priorizar variedad de actividades".

En tal sentido las debilidades referidas por los profesores y constatado por las autoras, constituyen temas a tener en cuenta para el rediseño del sistema de trabajo metodológico, teniendo en cuenta que la estrategia, los planes de trabajo metodológico y los planes individuales son flexibles y se ajustan a las necesidades de cada docente.

Al considerar los criterios de los profesores, los controles efectuados y el análisis de documentos podemos considerar que como resultado de la labor ejecutada se aprecian avances, tales como:

- La participación de los profesores a tiempo parcial en las actividades planificadas, a partir de ajustes en los horarios para su realización.

- Avances en el dominio de la estructura metodológica de la clase encuentro y en su ejecución.

- Fortalecimiento de la vinculación entre el personal docente de centros educativos de la localidad con los profesores del CUM que contribuya a su formación continua y como resultado en la formación integral del profesional.

- Se fortalece la preparación de los profesores en el conocimiento de su realidad que favorece el desarrollo de un proceso docente educativo que responde a las exigencias sociales.

- Perfeccionamiento en la orientación del estudio independiente en función del desarrollo de habilidades y estrategias necesarias para el autoaprendizaje en la modalidad semipresencial.

- Se han incrementado los espacios de comunicación e intercambio entre los profesores de la carrera, la disciplina y el año académico.

- Resultados docentes superiores en 20,8\% en el curso 2017-2018 con respecto al anterior.

- Satisfacción de los profesores con el trabajo metodológico desarrollado y de los estudiantes con la calidad de las actividades docentes.

- Participación y premiación de profesores en eventos científicos metodológicos. En los últimos dos cursos se han presentado 37 trabajos en seminario científico metodológico, de ellos 23 en conferencia científico metodológica.

- Participación de estudiantes en eventos científicos y jornadas científico estudiantil: 41 trabajos presentados por 85 estudiantes, autores y coautores, en el período 2016-2018.

El trabajo metodológico en el CUM, en particular en la formación de los formadores de formadores, comprende todo un conjunto de procesos a través de los cuales los profesores aprenden a analizar, a reflexionar, perfeccionan su práctica pedagógica para dar respuesta a las interrogantes profesionales y a la formación de un educador integral acorde con las demandas de la sociedad.

\section{Conclusiones}

En el presente trabajo se han presentado reflexiones y experiencias como resultado de la aplicación de la estrategia dirigida a la planificación, ejecución, control y evaluación del trabajo metodológico en el Centro Universitario Municipal de Jagüey Grande.

El estudio de documentos aprobados en eventos, de debates relacionados con la educación y de resultados de investigaciones, revela la necesidad de garantizar la formación continua de los profesores con el propósito de perfeccionar su práctica pedagógica acorde con la celeridad en la producción de conocimientos, el desarrollo científico tecnológico y las demandas sociales. Constituye una prioridad el trabajo metodológico como parte de la formación continua de los profesores de forma general y, en especial, de los que forman educadores para alcanzar la calidad y la pertinencia social de las instituciones docentes de la Educación Superior cubana e implica organizar espacios de intercambio reflexivo, a partir de considerarlo como proceso influenciado, mediado y condicionado por el contexto.

Resumiendo, las reflexiones y experiencias presentadas ponen de manifiesto que para el desarrollo del sistema de trabajo metodológico en el CUM se requiere considerar particularidades, tales como: el modelo pedagógico semipresencial, características del claustro, heterogeneidad de los estudiantes, el nexo estrecho con los diferentes niveles de dirección y organizativos de la Universidad de Matanzas, unido a la necesidad de formar profesionales comprometidos con el desarrollo local, han obligado a reflexionar en lo realizado tradicionalmente en cuanto a la preparación del profesor y cómo podría perfeccionarse.

Finalmente, buscando la conciliación entre lo reglamentado y las particularidades del CUM, se ha procedido, en primer lugar, a la realización del diagnóstico particularizado y continuo que ha garantizado la determinación de fortalezas y debilidades como punto de partida para la proyección de acciones y actividades, tanto individuales como colectivas, que posibilitan la participación de los profesores, a tiempo completo y a tiempo parcial, acorde con sus necesidades. En segundo lugar, el estrecho vínculo con los centros educativos donde los estudiantes desarrollan su labor docente ha dotado al profesor de 
Revista de la Escuela de Ciencias de la Educación, año 15, NRO. 14, vol. 2, JUlio a diciembre de 2019. PÁGinas 41-50. ISSN 1851-6297 (DESDE DICIEMBRE DE 2006 A DICIEMBRE DE 2017). ISSN 2362-3349 (EN LíNEA). El TRABAJO METOdOLÓGICO EN EL CENTRO UNIVERSITARIO

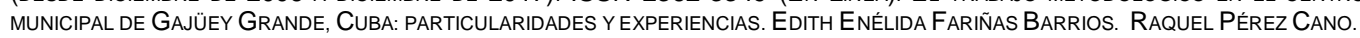

conocimientos y habilidades que inciden en su mejor desempeño. En tercer lugar, el aprendizaje colaborativo y la reflexión del profesor sobre su práctica pedagógica resulta necesario en aras de contribuir al desarrollo de su pensamiento crítico.

\section{Referencias bibliográficas}

- Alarcón, R. (2014). Universidad socialmente responsable. Conferencia inaugural 9no Congreso Internacional de Educación Superior Universidad 2014. La Habana, Cuba.

- Alarcón, R. (2015). Las ciencias de la educación en una universidad integrada e innovadora. Conferencia Congreso Internacional Pedagogía 2015. La Habana, Cuba.

- Amaro, M. C; Méndez, J. M. \& Mendoza, F. (2015). Un estudio de las características profesionales del docente universitario para atender a la diversidad. Revista Latinoamericana de Educación Inclusiva, Vol 8(2). pp.199 216.

- Fraga, E.; Milán, M.R. \& Echeverría, M. (2016). El trabajo metodológico en los departamentos de las universidades. X Taller Internacional de Pedagogía de la Educación Superior. Taller llevado a cabo en el $10^{\mathrm{mo}}$ Congreso Internacional de Educación Superior Universidad 2016. La Habana, Cuba.

- Horruitiner, P. (2006). La Universidad Cubana: el modelo de formación. La Habana: Editorial Félix Varela.

- Martín, D.R. (2015). La formación docente universitaria en Cuba: Sus fundamentos desde una perspectiva desarrolladora del aprendizaje y la enseñanza. Revista Estudios pedagógicos, Vol. 41(1). pp. 337-349.

- MES (2018). Resolución No. 2/2018. Reglamento de trabajo docente y metodológico de la educación superior. La Habana, Cuba.

- Miqueli, B. \& Polo, M. D. (2017). El trabajo metodológico en el año académico: necesidad para elevar la calidad de la clase en la educación Superior. Cooperación Universidad - Sociedad, Vol. 2 (2). pp. 42 - 48.

- Nava, N.G. \& Reynoso, J. (2015). Conceptualización y reflexión sobre la práctica educativa en un programa de formación contínua para docentes de educación media superior en México, Revista Educación. Vol.39(1), pp. 137-157.

- Núñez, J. \& Alcázar, A. (2016). Universidad y desarrollo local: contribuciones latinoamericanas. (Núñez y Alcázar, coord.) México: Coeditado por la Unión de Universidades de América Latina y el Caribe, el Ministerio de Educación Superior de Cuba y la Cátedra Ciencia, Tecnología, Sociedad e Innovación de la Universidad de La Habana.

- Orealc/Unesco. (2017). Declaración de Buenos Aires. "E 2030: Educación y Habilidades para el siglo 21". Aprobado en Reunión Regional de Ministros de Educación de América Latina y el Caribe. Buenos Aires, Argentina. Consultado el 7 de enero de 2018 en: http://unesdoc.unesco.org/images/0024/002472/247286S.pdf

- Pichs, B.; Hernández, D. \& Benítez, F. (2006). La educación en la diversidad desde la universalización de la universidad cubana. En Norma Castillo y Rosa O. Alfonso (Editoras). La nueva universidad cubana y su contribución a la universalización del conocimiento. La Habana, Cuba: Editorial Félix Varela.

- Rodríguez., Fariñas, E. y Pérez, R. (2015). Acciones para la superación del claustro de profesores de la Filial Universitaria Municipal de Jagüey Grande, Cuba. Sus resultados, pp. 231-248 en Revista de la Escuela de Ciencias de la Educación, año 11, número 10.

- Saborido, J.R. (2018). La universidad y la agenda 2030 de desarrollo sostenible en el centenario de la Reforma Universitaria de Córdoba. Visión desde Cuba. La Habana, Cuba: Editorial Félix Varela.

- Unesco (1998). Declaración mundial sobre la educación superior en el siglo XXI: visión y acción y marco de acción prioritaria para el cambio y el desarrollo de la educación superior. París. Octubre. Aprobado Conferencia Mundial sobre la Educación Superior La educación superior en el siglo XXI: Visión y acción 9 de octubre de 1998. Consultado el 5 de octubre de 2016 en:

http://www.unesco.org/ education/educprog/wche/declaration_spa.htm

- Vázquez-Cano, E. (2015). El reto de la formación docente para el uso de dispositivos digitales móviles en la educación superior. Perspectiva Educacional, Formación de Profesores, 54 (1), pp.149-162. 